\title{
OPTIMAL SHAPE DESIGN FOR A NOZZLE PROBLEM
}

\author{
R. BUTT ${ }^{1}$ \\ (Received 14 June 1991; revised 11 March 1992)
}

\begin{abstract}
In this paper, a gradient method is developed for the optimal shape design in a nozzle problem described by variational inequalities. It is known that this method can be used for the optimal shape design for systems described by partial differential equations (Pironneau [6]); it is used here for differential inequalities by taking limits of the expression resulting from an approximations scheme. The computations are done by the finite element method; the gradient of the criteria as a function of the coordinates nodes is computed, and the performance criterion is then minimised by the gradient method.
\end{abstract}

\section{Introduction}

The optimal shape problem can be solved for systems described by differential equations (Pironneau [6]). The purpose of this paper is to develop an optimal shape design for a nozzle problem described by a variational inequality.

Let $\Omega$ be a given domain and $D$ be any fixed domain which is contained in $\omega ; \partial \Omega$ is the boundary of the domain $\Omega$. The velocity $u(x)$ at a point $x$ in a nonviscous incompressible potential flow (such as for air or water at moderate speed) may be approximated by

$$
u(x)=\nabla \varphi(x) \quad x \in \Omega,
$$

'Centre for Advanced Studies in Pure \& Appl. Maths, Bahauddin Zakaryia University, Pakistan. (C) Australian Mathematical Society, 1993, Serial-fee code 0334-2700/93 


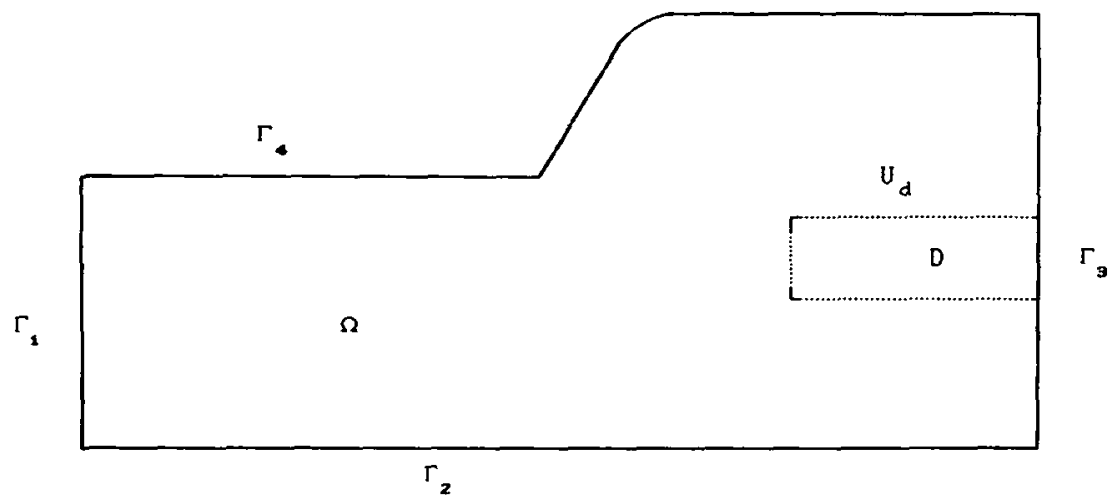

FIGURE 1. Physical set-up of the problem with domain $\Omega$, subdomain $D$ (i.e., $D \subset \Omega$ ), boundary of the domain $\partial \Omega=\bigcup_{i=1}^{4} \Gamma_{i}$ and velocity near the exit $U_{d}$.

where $\varphi$ satisfies a second-order partial differential equation on $\Omega$,

$$
-\nabla^{2} \varphi=f \text { in } \Omega .
$$

Then the flow in a nozzle $\Omega$ (the region occupied by the fluid) with a prescribed pressure drop $\varphi_{\Gamma_{1}}-\varphi_{\Gamma_{3}}$ is obtained by solving (1.2) with boundary conditions

$$
\partial \varphi /\left.\partial n\right|_{\Gamma_{2} \cup \Gamma_{4}}=0,\left.\quad \varphi\right|_{\Gamma_{1} \cup \Gamma_{3}}=0
$$

where $\partial \Omega=\cup \Gamma_{i}, i=1,2,3,4$. The physical set-up is depicted schematically in Figure 1. Consider the Sobolev space

$$
H_{0}^{1}(\Omega)=\left\{\phi \mid \phi \in H^{1}(\Omega), \quad \phi=0, \text { on } \partial \Omega\right\},
$$

where $H^{1}(\Omega)$ is the set of square integrable functions with square integrable first derivatives. Define the inner products (., .) and $a(.,$.$) on L^{2}(\Omega)$ and $H_{0}^{1}(\Omega)$ respectively, by

$$
(f, g)=\int_{\Omega} f g d x \quad \forall f, g \in L^{2}(\Omega)
$$

and

$$
a(\varphi, \phi)=\int_{\Omega} \nabla \varphi \cdot \nabla \phi d x \quad \forall \varphi, \phi \in H_{0}^{1}(\Omega),
$$

with the associated norms being denoted by $|f|^{2}=(f, f)$ and $\|\varphi\|^{2}=a(\varphi, \varphi)$. 
The problem we want to consider consists of finding the solution $\varphi$ so that

$$
\varphi \in H_{0}^{1}(\Omega),
$$

and

$$
a(\varphi, \phi-\varphi) \geq(f, \phi-\varphi) \quad \forall \phi \in H_{0}^{1}(\Omega) .
$$

We note that the bilinear form $a(.,$.$) in (1.5) is elliptic, i.e.,$

$$
a(\phi, \phi) \geq \alpha\|\phi\|^{2} \quad \alpha>0, \quad \forall \phi \in H_{0}^{1}(\Omega) .
$$

In our problem, we are interested in designing a nozzle that gives a prescribed velocity $U_{d}$ under the exit, say in some given domain $D$ which is a subset of the domain $\Omega$. To obtain an approximate design we shall solve the following optimisation problem:

$$
\min _{\Omega \in \theta} E(\Omega)=\int_{D}\left|\nabla \varphi(\Omega)-U_{d}\right|^{2} d x,
$$

where $\theta=\left\{\Omega: \Omega \supset D, ; \Gamma_{1}, \Gamma_{2}, \Gamma_{3}\right.$ are fixed, $\Gamma_{4}$ is any curve $\}$.

The optimal shape problem can be solved for the systems described by differential equations (Angrand [1]). So to solve the problem for the systems described by a differential inequality we shall introduce the first penalised equation,

$$
A \varphi_{\varepsilon}+(1 / \varepsilon) \varphi_{\varepsilon}^{-}=f, \quad \varphi_{\varepsilon} \in H_{0}^{1}(\Omega),
$$

where $V^{-}=-\sup (-V, 0)$, and $A: V=H_{0}^{1} \rightarrow V^{\prime}$ is a linear continuous and symmetric operator satisfying the coercivity condition, i.e. $(A \phi, \phi)=$ $a(\phi, \phi) \geq \alpha\|\phi\|^{2}$, for all $\phi \in V, \alpha>0$, and $A=-\nabla . \nabla$, whose solution $\varphi_{\varepsilon}$ tends to the solution of (1.5) when $\varepsilon \rightarrow 0$. For the existence and uniqueness of a solution of this equation, see Lions [5].

\section{Discretisation and optimisation}

We briefly review the method of finite elements. To illustrate the method, let (1.8) be discretised by triangulation elements of degree $m$. In variational form, (1.8) becomes: seek $\varphi_{\varepsilon} \in H_{0}^{1}(\Omega)$ so that

$$
\int_{\Omega}\left(\nabla \varphi_{\varepsilon} \cdot \nabla \omega+F\left(\varphi_{\varepsilon}\right) \omega-f \omega\right) d x=0, \quad \forall \omega \in H_{0}^{1}(\Omega),
$$


where $F\left(\varphi_{\varepsilon}\right)=(1 / \varepsilon) \varphi_{\varepsilon}^{-}$.

Let $\tau_{h}$ be a triangulation of $\Omega$, and $T_{k}$ is called the triangle, $\cup T_{k}=\Omega_{h} \subset \Omega$. The parameter $h$ is the size of the largest side or edge, and we assume that we have a family of triangulations of $\tau_{h}$. Let $P^{m}$ be the space of polynomials of degree $m$ on $\Omega_{h}$, and denote by

$$
H_{h}^{m}\left(\Omega_{h}\right)=\left\{\omega_{h} \in C^{0}\left(\Omega_{h}\right):\left.\omega_{h}\right|_{T_{k}} \in P^{m} \quad \forall T_{k} \in \tau_{h}\right\},
$$

the space of continuous piecewise polynomial functions on $\Omega_{h}$ (Pironneau [6]).

It is well known (see Ciarlet [3]) that $H_{h}^{m}\left(\Omega_{h}\right)$ is of finite dimension; then

$$
\int_{\Omega_{h}}\left(\nabla \varphi_{h, \varepsilon} \cdot \nabla \omega_{h}+F\left(\varphi_{h, \varepsilon}\right) \omega_{h}-f \Omega_{h}\right) d x=0
$$

reduces to the solution of a linear symmetric positive definite system plus the numerical computation of some integrals. More precisely, if $\left\{\omega^{i}\right\}_{i}^{N}$ is a basis for $H_{h}^{m}\left(\Omega_{h}\right),(2.3)$ is equivalent to (Pironneau [6])

$$
\widehat{A} \varphi=F,
$$

where $\widehat{A}_{i j}=\int_{\Omega_{h}}\left(\nabla \omega^{i} \cdot \nabla \omega^{j}+F\left(\varphi_{h, \varepsilon}\right) \omega^{j}\right) d x ; \quad F_{i}=\int_{\Omega_{h}} f \omega^{i} d x$

$$
\varphi_{h}=\sum_{i=1}^{N} \varphi_{i} \omega^{i} .
$$

The $\left\{\omega^{i}\right\}$ are polynomials of degree $\leq m$ on $T_{k}$, so $\widehat{A}_{i j}$ can be computed exactly. In the case $m=1$, if $\left\{q^{j}\right\}_{i}^{N}$ denote the vertices of $\tau_{h}$, then $\left\{\omega^{i}\right\}$ are uniquely determined by

$$
\omega^{i}\left(q^{j}\right)=\delta_{i j} \quad \forall i, j=1, \ldots, N .
$$

In the case $m=2$, if $\left\{q^{j k}\right\}$ denote the middles of the sides of vertices $\left\{q^{j}, q^{k}\right\}$, then $\left\{\omega^{i}\right\}$ is uniquely determined by

$$
\omega^{i}\left(q^{j}\right)=\delta_{i j} \quad \forall i, j=\left\{1, \ldots, N^{\prime}\right\} \cup\left(\left\{1, \ldots, N^{\prime}\right\} \times\left\{1, \ldots, N^{\prime}\right\}\right) .
$$

It is possible to consider our optimisation problem in this new setting. The optimal shape will be found by successive approximation starting with an initial guess $\Omega_{h}^{0}$, and the algorithm is then developed by means of a gradient method. We note that the problem has been discretised, so that the shape $\Omega_{h}$ is defined by the coordinates of the nodes. The expression for the cost function $E$ is now

$$
E\left(\Omega_{h}\right)=\int_{D_{h}}\left(\nabla \varphi_{h, \varepsilon}-U_{d, h}\right)^{2} d x
$$


where $\varphi_{h . \varepsilon}$ is the solution of the differential equation (2.3) on $\Omega_{h}$ and $U_{d, h}$ and $D_{h}$ are the approximations of $U_{d}$ and $D$ respectively. The following theorem has been adapted from Pironneau [6] to compute the gradient of the cost function $E$ at $\Omega_{h}$. For the proof of this theorem see Butt [2].

THEOREM 1. If $E$ is given by (2.6) and $\varphi_{h, \varepsilon}$ by (2.3), then

$$
\begin{aligned}
& \partial E / \partial q_{l}^{k}= \int_{\Omega_{h}} \partial / \partial x_{l}\left\{\omega^{k}\left(\nabla \varphi_{h, \varepsilon}-U_{d, h}\right)^{2}\right\} d x \\
&+\int_{\Omega_{h}}\left\{\left(\nabla \varphi_{h, \varepsilon} \cdot \nabla \omega^{k}\right) \partial P_{h, \varepsilon} / \partial x_{l}-\left(\nabla \varphi_{h, \varepsilon} \cdot \nabla P_{h, \varepsilon}\right) \partial \omega^{k} / \partial x_{l}\right\} d x \\
&+\int_{\Omega_{h}}\left\{\partial / \partial x_{l}\left(f P_{h, \varepsilon} \omega^{k}\right)-\left(f \omega^{k} \partial P_{h, \varepsilon} / \partial x_{l}\right)\right\} d x \\
&+\int_{\Omega_{h}}\left\{\left(F\left(\varphi_{h, \varepsilon}\right) \nabla \omega^{k} \partial P_{h, \varepsilon} / \partial x_{l}\right)-\partial / \partial x_{l}\left(\omega^{k} F\left(\varphi_{h, \varepsilon}\right) P_{h, \varepsilon}\right)\right\} d x, \\
& l=1,2 \text { and } k=1, \ldots, n, \quad q^{k} \in D_{h},
\end{aligned}
$$

where

$$
P_{h, \varepsilon} \in H_{h}^{1}\left(\Omega_{h}\right)
$$

is the solution of

$$
\begin{aligned}
& \int_{\Omega_{h}}\left(\nabla P_{h, \varepsilon} \cdot \nabla \omega_{h}+F^{\prime}\left(\varphi_{h, \varepsilon}\right) P_{h, \varepsilon} \omega_{h}\right) d x \\
& \left.=2 \int_{D_{h}}\left(\nabla \varphi_{h, \varepsilon}\right)-U_{d, h}\right) \nabla \omega_{h} d x, \quad \omega_{h} \in H_{0}^{1}\left(\Omega_{h}\right)
\end{aligned}
$$

with $F^{\prime}\left(\varphi_{h, \varepsilon}\right)=(1 / \varepsilon) d / d \varphi\left(\varphi_{h, \varepsilon}^{-}\right)$, and $(2.8)$ is equivalent to the second penalised equation,

$$
A P_{h, \varepsilon}+F^{\prime}\left(\varphi_{h, \varepsilon}\right) P_{h, \varepsilon}=-2 \nabla\left(\nabla \varphi_{h, \varepsilon}-U_{d, h}\right)=f_{1} .
$$

We note that the function $\varphi_{h, \varepsilon} \rightarrow \varphi_{h, \varepsilon}^{-}$is not differentiable at $\varphi_{h, \varepsilon}=0$; we have defined $F^{\prime}(0)=0$. This choice turns out to be unimportant because $f_{h, \varepsilon}>0$, on $\Omega_{h}$, with exception of a (zero-measure) subset of $\partial \Omega_{h}$. For more details see Butt [2], where an approximation scheme is introduced for proving this.

\section{Optimal shape design for a variational inequality}

Now we come to the implementation of the main idea of our treatment, that is, to take the limit as $\varepsilon$ tends to zero of these quantities. First we shall find the 
value of the limit of the cost function $E$, as $\varepsilon$ tends to zero. Since we know (Lions [5]) that

$$
\varphi_{h, \varepsilon} \rightarrow \varphi_{h} \text { in } H_{h}^{1}\left(\Omega_{h}\right) \text { weakly as } \varepsilon \rightarrow 0
$$

and also,

$$
\varphi_{h, \varepsilon} \rightarrow \varphi_{h} \text { in } L^{2}\left(\Omega_{h}\right) \text { strongly as } \varepsilon \rightarrow 0,
$$

by taking the limit (as $\varepsilon \rightarrow 0$ ), on both sides of (2.6), we obtain:

$$
\lim _{\varepsilon \rightarrow 0} E\left(\Omega_{h}\right)=\lim _{\varepsilon \rightarrow 0} \int_{D_{h}}\left(\nabla \varphi_{h, \varepsilon}-U_{d, h}\right)^{2} d x
$$

Since $\varphi_{h, \varepsilon} \rightarrow \varphi_{h}$ in $L^{2}\left(\Omega_{h}\right)$ strongly, so (Glowinski et al. [4])

$$
\lim _{\varepsilon \rightarrow 0} \int_{D_{h}}\left(\nabla \varphi_{h, \varepsilon}-U_{d, h}\right)^{2} d x \rightarrow \int_{D_{h}}\left(\nabla \varphi_{h}-U_{d, h}\right)^{2} d x
$$

since the functional $\varphi_{h} \rightarrow \int_{D_{h}}\left(\nabla \varphi_{h}-U_{d, h}\right)^{2} d x$ is continuous in $L^{2}\left(\Omega_{h}\right)$; so the above equation becomes:

$$
E\left(\Omega_{h}\right)=\int_{D_{h}}\left(\nabla \varphi_{h}-U_{d, h}\right)^{2} d x
$$

which is the required value of the cost function $E$ as $\varepsilon$ tends to zero. Now we shall find the value of the gradient of the cost function (2.7) as $\varepsilon$ tends to zero:

$$
\begin{aligned}
\lim _{\varepsilon \rightarrow 0} \partial E / \partial q_{l}^{k}=\lim _{\varepsilon \rightarrow 0}\left[\int_{\Omega_{h}} \partial / \partial x_{l}\left\{\omega^{k}\left(\nabla \varphi_{h, \varepsilon}-U_{d, h}\right)^{2}\right\} d x\right. \\
+\int_{\Omega_{h}}\left\{\left(\nabla \varphi_{h, \varepsilon} \cdot \nabla \omega^{k}\right) \partial P_{h, \varepsilon} / \partial x_{l}-\left(\nabla \varphi_{h, \varepsilon} \cdot \nabla P_{h, \varepsilon}\right) \partial \omega^{k} / \partial x_{l}\right\} d x \\
+\int_{\Omega_{h}}\left\{\partial / \partial x_{l}\left(f P_{h, \varepsilon} \omega^{k}\right)-\left(f \omega^{k} \partial P_{h, \varepsilon} / \partial x_{l}\right)\right\} d x \\
+\int_{\Omega_{h}}\left\{\left(F\left(\varphi_{h, \varepsilon}\right) \nabla \omega^{k}\left(\partial P_{h, \varepsilon} / \partial x_{l}\right)-\partial / \partial x_{l}\left(\omega^{k} F\left(\varphi_{h, \varepsilon}\right) P_{h, \varepsilon}\right)\right\} d x\right] .
\end{aligned}
$$

Now we need to find the limit of the vector $P_{h, \varepsilon}$ as $\varepsilon$ tends to zero; in the Appendix we prove the following theorem which shows that this limit, $P_{h}$, is itself the solution of a variational inequality. 
THEOREM 2. As $\varepsilon \rightarrow 0, P_{h, \varepsilon} \rightarrow P_{h}$ in $H_{0}^{1}\left(\Omega_{h}\right), P_{h}$ being the solution of the variational inequality

$$
a\left(P_{h}, \omega_{h}-P_{h}\right) \geq\left(f_{1}, \omega_{h}-P_{h}\right), \quad \forall \omega_{h} \in H_{0}^{1}\left(\Omega_{h}\right)
$$

where $f_{1}$ is defined by (2.9).

The proof of Theorem 2 has been explained in detail in the appendix. Now we shall compute the gradient of the cost function when $\varepsilon$ tends to zero, by using (3.3).

Since we know (Glowinski et al. [4]) that

$$
\lim _{\varepsilon \rightarrow 0} \int_{\Omega_{h}}\left(\nabla P_{h, \varepsilon} \cdot \nabla \varphi_{h, \varepsilon}\right) d x=\int_{\Omega_{h}}\left(\nabla P_{h} \cdot \nabla \varphi_{h}\right) d x,
$$

and

$$
\partial / \partial x_{1}\left(\varphi_{h, \varepsilon}\right) \rightarrow \partial / \partial x_{1}\left(\varphi_{h}\right) \text { in } L^{2}\left(\Omega_{h}\right) \text { weakly, as } \varepsilon \rightarrow 0 \text {, }
$$

then $\varphi_{h, \varepsilon} \rightarrow \varphi_{h}$ in $L^{2}\left(\Omega_{h}\right)$ strongly as $\varepsilon \rightarrow 0$; so (3.3) gives rise to

$$
\begin{aligned}
& \partial E / \partial q_{l}^{k}= \int_{\Omega_{h}}\left(\nabla \varphi_{h}-U_{d, h}\right)^{2} \partial / \partial x_{l}\left(\omega^{k}\right) d x \\
&+ \int_{\Omega_{h}}\left\{\left(\nabla \varphi_{h} \cdot \nabla \partial / \partial x_{l}\left(P_{h}\right)\right) \omega^{k}\right. \\
&\left.+\left(\nabla \varphi_{h} \cdot \nabla \partial / \partial x_{l}\left(\omega^{k}\right)\right) P_{h}-\left(\nabla \varphi_{h} \cdot \nabla \partial / \partial x_{l}\left(P_{h}\right)\right) \nabla \omega^{k}\right\} d x \\
&+ \int_{\Omega_{h}}\left\{2\left(\nabla \varphi_{h} \cdot \nabla \omega^{k}\right) \partial / \partial x_{l}\left(P_{h}\right)\right. \\
&\left.+\left(\nabla P_{h} \cdot \nabla \omega^{k}\right) \partial / \partial x_{l}\left(\varphi_{h}\right)-f \omega^{k} \partial / \partial x_{l}\left(P_{h}\right)\right\} d x \\
&- \int_{\Omega_{h}}\left\{\left(\nabla \varphi_{h} \cdot \nabla \nabla \omega^{k}\right) \partial / \partial x_{l}\left(P_{h}\right)\right. \\
&\left.-\left(\nabla P_{h} \cdot \nabla \partial / \partial x_{l}\left(\varphi_{h}\right)\right) \omega^{k}-f \nabla \omega^{k} \partial / \partial x_{l}\left(P_{h}\right)\right\} d x \\
& l=1,2, \quad \text { and } k=1, \ldots, n
\end{aligned}
$$

where $\varphi_{h}$ is the solution of (1.5) and $P_{h}$ is the solution of (3.4).

We define then an algorithm to solve the optimal shape problem for the systems described by a differential inequality, i.e. when $\varepsilon$ tends to zero, and in this algorithm it has been found necessary to use a second-order approximation, that is, $m=2$, which made us able to compute (3.5).

ALGORITHM

1. Choose $\Omega_{h}^{0}$, i.e., $\left\{q^{k, 0}\right\}$.

2. Compute $\varphi_{h}^{m^{\prime}}$ (with $m=2$ ).

3. Compute $P_{h}^{m^{\prime}}$. 
4. Compute $G_{l}^{k}=-\partial E / \partial q_{l}^{k}, l=1,2$, and $k=1, \ldots, n, q^{k} \notin D_{h}$.

5. Let $q^{k, m^{\prime}}(\rho)=q^{k, m^{\prime}}+\rho G_{l}^{k}$. Compute $\rho^{m^{\prime}}$, an approximation of arg $\min _{0<\rho<\rho_{\max }} E\left(\left\{q^{k, m^{\prime}}(\rho)\right\}\right)$, where $E$ is given by (3.2).

6. Set $q^{k, m^{\prime}+1}=q^{k, m^{\prime}}(\rho)$.

7. Perform a terminal check, if necessary go on with the same procedure in $q^{k, m^{\prime}+1}$, i.e. go back to 1 .

\section{Description of the program and algorithms used}

The implementation of an algorithm $(m=2)$ will be described here. The optimum design program is composed of the following modules.

MODULE 1. A module for solving the direct problem (or state problem). Find $\varphi_{h} \in H_{h}^{2}\left(\Omega_{h}\right)$ such that

$$
\int_{\Omega_{h}}\left(\nabla \varphi_{h} \cdot \nabla \omega_{h}-f \omega_{h}\right) d x \geq 0 \quad \forall \omega_{h} \in H_{h}^{2}\left(\Omega_{h}\right)
$$

or, find $\varphi_{h} \in H_{h}^{2}\left(\Omega_{h}\right)$ such that

$$
I\left(\varphi_{h}\right) \leq I\left(\omega_{h}\right) \quad \forall \omega_{h} \in H_{h}^{2}\left(\Omega_{h}\right),
$$

where $I\left(\varphi_{h}\right)$ is defined as follows:

$$
I\left(\varphi_{h}\right)=1 / 2 \int_{\Omega_{h}}\left|\nabla \varphi_{h}\right|^{2} d x-\int_{\Omega_{h}} f \varphi_{h} d x
$$

minimised over the convex set $K_{1}=\left\{\psi_{h} \in H^{2}\left(\Omega_{h}\right), \psi_{h} \geq 0\right.$ a.e. in $\left.\Omega_{h}\right\}$, where $\varphi_{h}$ is the solution of (4.1). The method used for the minimisation of this functional will be explained briefly. The function $I\left(\varphi_{h}\right)$ may be written $I\left(\varphi_{1}, \ldots, \varphi_{N(h)}\right)$ to emphasise the dependence of $\varphi_{h}$ on the coefficients in (2.5). The problem (4.2) is solved by the relaxation method, with

$$
\varphi_{h}^{0}=\left(\varphi_{1}^{0}, \ldots, \varphi_{N h}^{0}\right) \text { given in } H_{h}^{2}\left(\Omega_{h}\right),
$$

with $\varphi_{h}^{n}$ known, then $\varphi_{h}^{n+1}$ is determined coordinate by coordinate, further iterations in the algorithm being given by

$$
\varphi^{n+1}=\varphi^{n}+\omega\left(\varphi^{n+1 / 2}-\varphi^{n}\right) .
$$




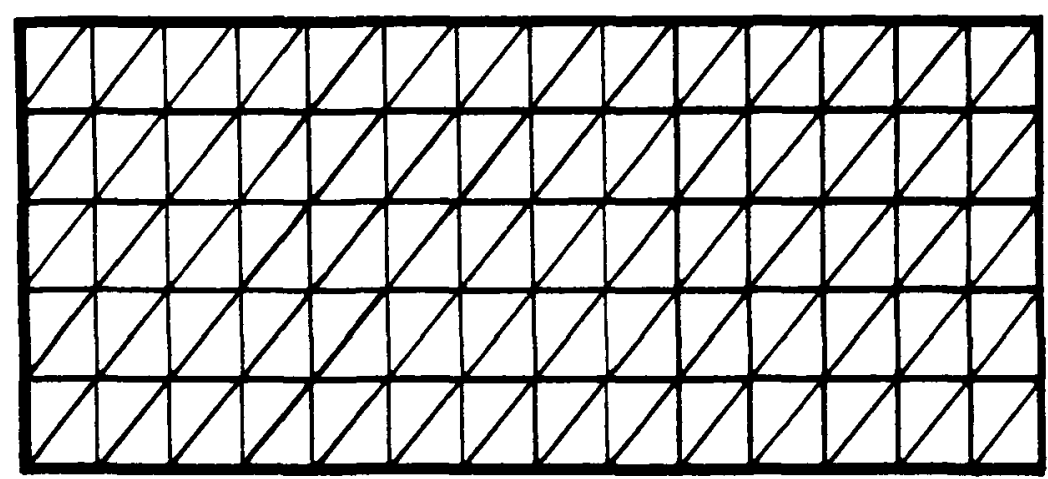

FIGURE 2. Indicates the initial shape with performance criterion $E\left(\Omega_{h}^{0}\right)=0.16452$ after iteration zero. The total number of nodes is 90 and the total number of triangles is 140 for the domain $\Omega$. For the subdomain $D$ the total number of nodes is 36 and the total number of triangles is 50 .

Here $\omega$ is the relaxation parameter, $0<\omega<2$. The process is stopped when

$$
\sum_{i=1}^{N h}\left|\varphi_{i}^{n+1}-\varphi_{i}^{n}\right| / \sum_{i=1}^{N h}\left|\varphi_{i}^{n+1}\right| \leq \varepsilon_{r} .
$$

(In our computational experiments we took $\varepsilon_{r}=10^{-5}$.)

MODULE 2. A module for solving the adjoint-state problem, whose solution is needed to compute the descent direction (the vector $G$ ). The adjoint state $P_{h} \in H_{h}^{2}\left(\Omega_{h}\right)$ given by the solution of the following variational inequality,

$$
\int_{\Omega_{h}}\left(\nabla P_{h} \cdot \nabla \omega_{h}\right) d x-\int_{D_{h}}\left(f_{1} \omega_{h}\right) d x \geq 0 \quad \forall \omega_{h} \in H_{h}^{2}\left(\Omega_{h}\right) .
$$

In the Appendix, we show that this variational inequality has a solution which minimises the following functional:

$$
I\left(P_{h}\right)=\frac{1}{2} \int_{\Omega_{h}}\left|\nabla P_{h}\right|^{2} d x-\int_{D_{h}} f_{1} P_{h} d x, \quad P_{h} \in H_{h}^{2}\left(\Omega_{h}\right)
$$

over the convex set $K_{1}$ and $P_{h}$ is the solution of (4.4), and $f_{1}$ is defined by (2.9). For this problem, we use the same optimisation method used in the case of the state problem.

MODULE 3. A module for the computation of the descent direction, i.e. the gradient of the cost function $E$ when we know the solution $\varphi_{h}$ of the state 


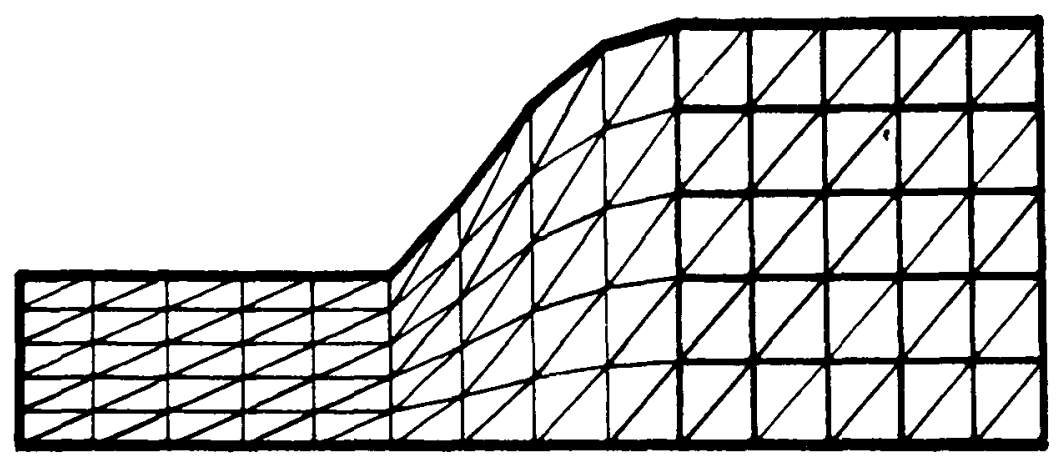

FIGURE 3. Indicates the new shape after 15 iterations with new performance criterion $E\left(\Omega_{h}^{15}\right)=$ 0.010921 . The total number of nodes is 90 and the total number of triangles is 140 for the domain $\Omega$. For the subdomain $D$ the total number of nodes is 36 and the total number of triangles is 50 .

problem and the solution $P_{h}$ of the adjoint state problem. In the formula we must account for the variability of the criterion domain.

MODULE 4. A module minimising the criterion functional when we know a descent direction. We used the gradient method with optimal choice of step length $\rho$ and eventually projection.

MODULE 5. A drawing module for the plotting of the results related to a given geometry. This is convenient for quickly analysing computational results.

The finite element method was used to solve (1.5), (3.4), and (3.5) with $f=0$ and $U_{d, h}=0.1$. The triangulation is composed of 90 nodes and 140 triangles for the domain $\Omega_{h}$ and 36 nodes and 50 triangles for the subdomain $D_{h}$. The initial shape of the problem is shown in Figure 2 and we can also see in Figure 2 the subdomain $D_{h}$ where the criterion $E$

$$
E\left(\Omega_{h}\right)=\int_{D_{h}}\left(\nabla \varphi_{h}-U_{d, h}\right)^{2} d x
$$

is evaluated. The starting value of the criterion is $E\left(\Omega_{h}^{0}\right)=0.16452$ with $U_{d, h}=0.1$ given, at iteration zero. The new shape of the problem is shown in Figure 3 after 15 iterations with criterion $E\left(\Omega_{h}^{15}\right)=0.010921$, and Figure 4 shows the final shape of the problem with criterion $E\left(\Omega_{h}^{27}\right)=0.000415$ after 24 iterations. Figure 5 shows the relation between the performance criterion $E$ and the number of iterations. 


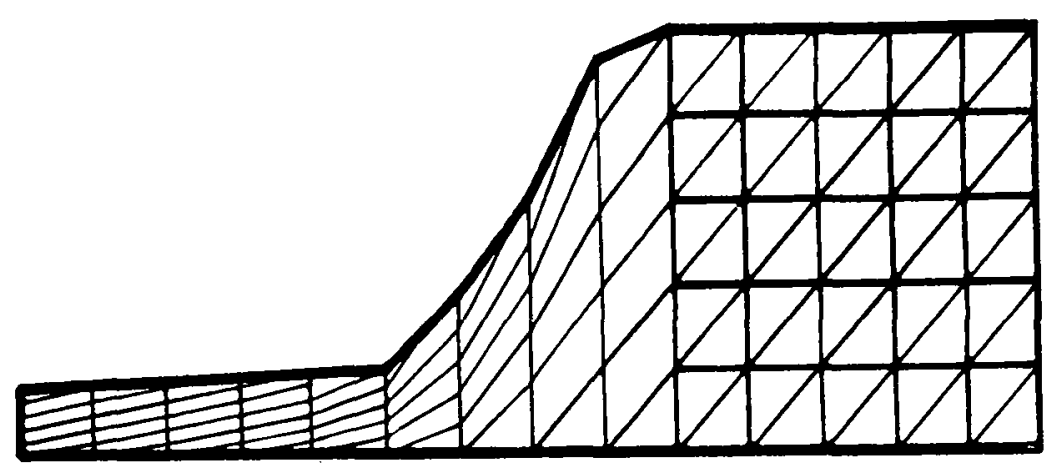

FIGURE 4. Indicates the final shape of the problem after 27 iterations with performance criterion $E\left(\Omega_{h}^{27}\right)=0.000415$. The total number of nodes is 90 and the total number of triangles is 140 for the domain $\Omega$. For the subdomain $D$ the total number of nodes is 36 and the total number of triangles is 50 .

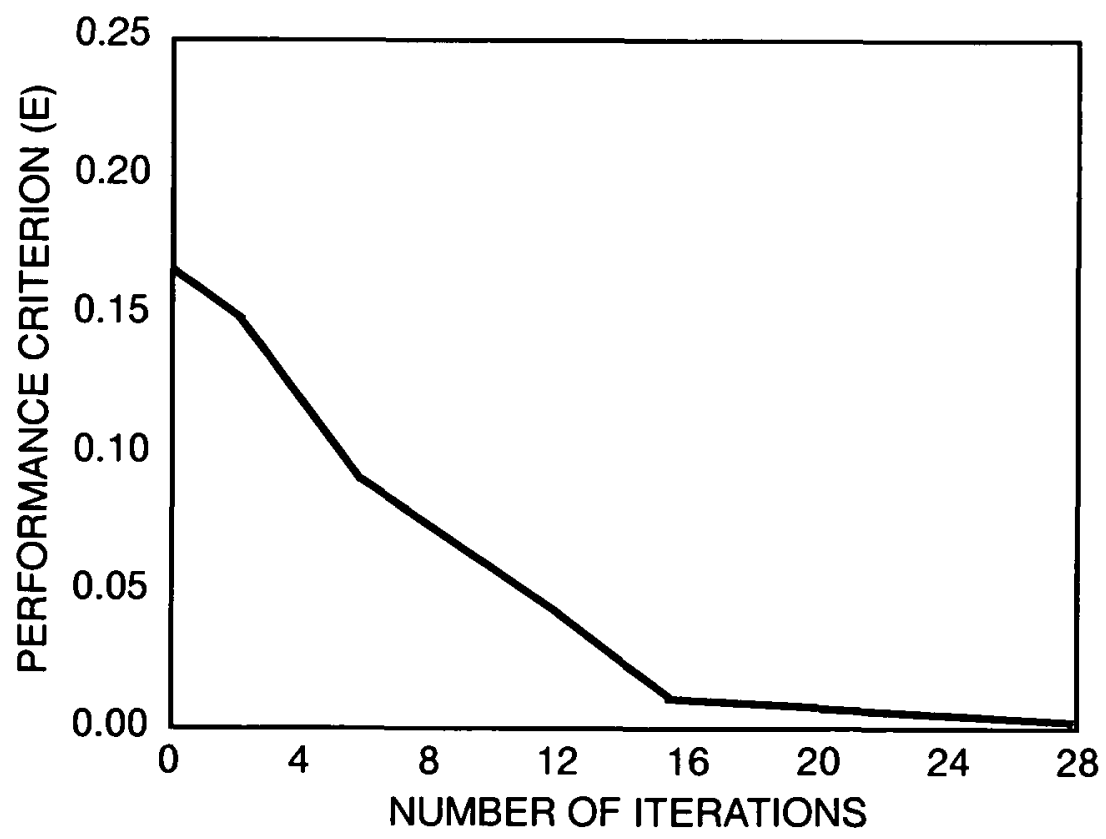

FIGURE 5: Indicates the relation between performance criterion and number of iterations. 


\section{Conclusions}

We have developed a method for the optimal shape design for the nozzle problem. The work has been helped by the fact that our system is governed by a variational inequality, with all its strong properties, which make the approximation and computation of solutions and optimal shapes that much simpler. The main theoretical result - Theorem 2 in Section 3 - shows that the vector which eventually defines the search direction for a minimum, is itself the solution of an associated variational inequality. The practical results consist of the development of a computationally-complex method for the determination of the optimal shapes, which can be adapted to other problems of current interest.

\section{Appendix}

The main purpose of this appendix is to sketch the proof of Theorem 2. We can prove that the functions $P_{h, \varepsilon}$ are non-negative on $\Omega_{h}$. Before proving Theorem 2, we prove the following lemma.

LEMMA 1. Let $a\left(P_{h, \varepsilon}, \phi_{h, \varepsilon}\right)$ be a bilinear, continuous form on $H_{h}^{2}\left(\Omega_{h}\right) \times H_{h}^{2}\left(\Omega_{h}\right)$ such that

$$
a\left(P_{h, \varepsilon}, P_{h, \varepsilon}\right) \geq 0 \quad \forall P_{h, \varepsilon} \in H_{h}^{2}\left(\Omega_{h}\right) .
$$

Then the function $\phi_{h, \varepsilon} \rightarrow a\left(\phi_{h, \varepsilon}, \phi_{h, \varepsilon}\right)$ is lower-semicontinuous with respect to the weak topology.

PROOF. From the bilinearity, we have for all $P_{h, \varepsilon} \in H_{h}^{2}\left(\Omega_{h}\right), \phi_{h, \varepsilon} \in H_{h}^{2}\left(\Omega_{h}\right)$

$$
\begin{aligned}
a\left(\phi_{h, \varepsilon}, \phi_{h, \varepsilon}\right)= & a\left(P_{h, \varepsilon}, P_{h, \varepsilon}\right)+\left[a\left(P_{h, \varepsilon}, \phi_{h, \varepsilon}-P_{h, \varepsilon}\right)\right. \\
& \left.+a\left(\phi_{h, \varepsilon}-P_{h, \varepsilon}, P_{h, \varepsilon}\right)+a\left(P_{h, \varepsilon}-\phi_{h, \varepsilon}, P_{h, \varepsilon}-\phi_{h, \varepsilon}\right)\right] .
\end{aligned}
$$

Now we use the condition of ellipticity, i.e.

$$
a\left(P_{h, \varepsilon}, P_{h, \varepsilon}\right) \geq 0
$$

which implies that

$$
a\left(\phi_{h, \varepsilon}, \phi_{h, \varepsilon}\right) \geq a\left(P_{h, \varepsilon}, P_{h, \varepsilon}\right)+\left[a\left(P_{h, \varepsilon}, \phi_{h, \varepsilon}-P_{h, \varepsilon}\right)+a\left(\phi_{h, \varepsilon}-P_{h, \varepsilon}, P_{h, \varepsilon}\right)\right] .
$$


Now, let $\phi_{h, \varepsilon} \rightarrow P_{h}$ in $H_{h}^{2}\left(\Omega_{h}\right)$ weakly; from the continuity of " $a$ ", and the fact that

$$
a\left(P_{h}, \phi_{h, \varepsilon}-P_{h}\right) \rightarrow 0 \quad \text { and } \quad a\left(\phi_{h, \varepsilon}-P_{h}, P_{h}\right) \rightarrow 0 \text {, }
$$

we have

$$
\liminf _{\phi_{h, \varepsilon} \rightarrow P_{h}} a\left(\phi_{h, \varepsilon}, \phi_{h, \varepsilon}\right) \geq a\left(P_{h}, P_{h}\right) .
$$

Hence the map $\phi_{h, \varepsilon} \rightarrow a\left(\phi_{h, \varepsilon}, \phi_{h, \varepsilon}\right)$ is weakly lower-semicontinuous.

In connection with the behaviour of the subsequence $P_{h, \varepsilon}$ as $\varepsilon \rightarrow 0$, we have THEOREM 2. As $\varepsilon \rightarrow 0, P_{h, \varepsilon} \rightarrow P_{h}$ in $H_{h}^{2}\left(\Omega_{h}\right), P_{h}$ being the solution of the variational inequality

$$
a\left(P_{h}, \omega_{h}-P_{h}\right) \geq\left(f_{1}, \omega_{h}-P_{h}\right) \quad \forall \omega_{h} \in H_{h}^{2}\left(\Omega_{h}\right),
$$

where $f_{1}$ is defined by (2.9).

PROOF. Consider the second penalised equation

$$
A P_{h, \varepsilon}+(1 / \varepsilon)\left(d / d \varphi\left(\varphi_{h, \varepsilon}^{-}\right)\right) P_{h, \varepsilon}=f_{1},
$$

or, in variational form,

$$
\int_{\Omega_{h}}\left(\left(\nabla P_{h, \varepsilon} \cdot \nabla \omega_{h}\right)+(1 / \varepsilon)\left(\left(d / d \varphi\left(\varphi_{h, \varepsilon}^{-}\right)\right) P_{h, \varepsilon}, \omega_{h}\right)\right) d x=\int_{D_{h}}\left(f_{1}, \omega_{h}\right) d x .
$$

With $P_{h, \varepsilon}=\omega_{h}$, we have

$$
\int_{\Omega_{h}}\left(\left(\nabla P_{h, \varepsilon} \cdot \nabla P_{h, \varepsilon}\right)+\left(F^{\prime}\left(\varphi_{h, \varepsilon}\right) P_{h, \varepsilon}, P_{h, \varepsilon}\right)\right) d x=\int_{D_{h}}\left(f_{1}, P_{h, \varepsilon}\right) d x,
$$

where $F^{\prime}\left(\varphi_{h, \varepsilon}\right)=(1 / \varepsilon)\left(d / d \varphi\left(\varphi_{h, \varepsilon}^{-}\right)\right) \geq 0$, and

$$
\int_{\Omega_{h}}\left(d / d \varphi\left(\varphi_{h, \varepsilon}^{-}\right)\right) P_{h, \varepsilon}^{2} d x \geq 0
$$

Then, by (1.6) and (7), we have

$$
0 \leq \alpha\left\|P_{h, \varepsilon}\right\|^{2} \leq \int_{\Omega_{h}}\left(\left(\nabla P_{h, \varepsilon} \cdot \nabla P_{h, \varepsilon}\right)+F^{\prime}\left(\varphi_{h, \varepsilon}\right) P_{h, \varepsilon}^{2}\right) d x=\int_{D_{h}}\left(f_{1}, P_{h, \varepsilon}\right) d x,
$$


or,

$$
\begin{aligned}
\alpha\left\|P_{h, \varepsilon}\right\|^{2} & \leq \int_{D_{h}}\left(f_{1}, P_{h, \varepsilon}\right) d x \leq\left\|f_{1}\right\|\left\|P_{h, \varepsilon}\right\|, \\
\alpha\left\|P_{h, \varepsilon}\right\|^{2} & \leq C^{1}\left\|P_{h, \varepsilon}\right\| \quad\left(C^{1}=\left\|f_{1}\right\|\right), \\
\left\|P_{h, \varepsilon}\right\| & \leq C_{11}, \quad\left(C_{11}=c^{1} / \alpha=\text { constant, independent of } \varepsilon\right) .
\end{aligned}
$$

A subsequence, also denoted by $P_{h, \varepsilon}$, can then be extracted from the sequence $P_{h, \varepsilon}$, such that

$$
P_{h, \varepsilon} \rightarrow P_{h} \text { weakly in } H_{h}^{2} .
$$

Since we have assumed that $P_{h, \varepsilon} \geq 0$ on $\Omega_{h}, P_{h} \geq 0$ on $\Omega_{h}$. By writing (7) in the following form:

$$
\begin{aligned}
a\left(P_{h, \varepsilon}, \omega_{h}-P_{h, \varepsilon}\right) & \\
-\left(f_{1}, \omega_{h}-P_{h, \varepsilon}\right) & =-\left(F^{\prime}\left(\varphi_{h, \varepsilon}\right) P_{h, \varepsilon}, \omega_{h}-P_{h, \varepsilon}\right) \\
& =\left(F^{\prime}\left(\varphi_{h, \varepsilon}\right) P_{h, \varepsilon}, P_{h, \varepsilon}\right)-\left(F^{\prime}\left(\varphi_{h, \varepsilon}\right) P_{h, \varepsilon}, \omega_{h}\right) \\
& =1 / \varepsilon\left[\left(\widehat{H} P_{h, \varepsilon}, P_{h, \varepsilon}\right)-\left(\widehat{H} P_{h, \varepsilon}, \omega_{h}\right)\right],
\end{aligned}
$$

where $\widehat{H} \equiv d / d \varphi\left(\varphi_{h, \varepsilon}^{-}\right)$. Consider now (9) only for those $\omega_{h}=W_{h} \in B \subset$ $H_{h}^{2}\left(\Omega_{h}\right)$, with $B$ the subset of the convex set $K_{1}$ composed of the basis elements for $H_{h}^{2}\left(\Omega_{h}\right)$. Now we shall prove that the right-hand side of (9) is positive, that is,

$$
\left(\widehat{H} P_{h, \varepsilon}, P_{h, \varepsilon}\right)>\left(\widehat{H} P_{h, \varepsilon}, W_{h}\right), \quad \text { provided } h \text { is sufficiently small. }
$$

Since $\widehat{H}$ is positive operator, $\left(\widehat{H} P_{h, \varepsilon}, P_{h, \varepsilon}\right)>0$; we can assume that $\left(\widehat{H} P_{h, \varepsilon}, W_{h}\right)$ $\geq 0$; otherwise (9) is automatically true. We can make the right-hand side of the inequality (10) as small as possible; note that $P_{h, \varepsilon}$ does not depend much on $h$ (from (4)), but that the support of $W_{h}$ can be made as small as possible by taking $h$ small enough, the maximum value of $W_{h}$ is of course 1 . Therefore from (10) we can see that, under these conditions,

$$
\begin{gathered}
a\left(P_{h, \varepsilon}, W_{h}-P_{h, \varepsilon}\right)-\left(f_{1}, W_{h}-P_{h, \varepsilon}\right) \\
=1 / \varepsilon\left[\left(\widehat{H} P_{h, \varepsilon}, P_{h, \varepsilon}\right)-\left(\widehat{H} P_{h, \varepsilon}, W_{h}\right)\right] \geq 0, \\
\text { for } W_{h} \in B \subset H_{h}^{2}\left(\Omega_{h}\right) .
\end{gathered}
$$

Hence (11) can be written as

$$
a\left(P_{h, \varepsilon}, W_{h}-P_{h, \varepsilon}\right)-\left(f_{1}, W_{h}-P_{h, \varepsilon}\right) \geq 0, \quad W_{h} \in B \subset H_{h}^{2}\left(\Omega_{h}\right)
$$


or

$$
a\left(P_{h, \varepsilon}, W_{h}\right)-\left(f_{1}, W_{h}-P_{h, \varepsilon}\right) \geq a\left(P_{h, \varepsilon}, P_{h, \varepsilon}\right) .
$$

Letting $\varepsilon \rightarrow 0$ in (12), we obtain

$$
a\left(P_{h}, W_{h}\right)-\left(f_{1}, W_{h}-P_{h}\right) \geq \liminf _{\varepsilon \rightarrow 0} a\left(P_{h, \varepsilon}, P_{h, \varepsilon}\right) .
$$

By applying the lemma, we obtain now

$$
\liminf _{\varepsilon \rightarrow 0} a\left(P_{h, \varepsilon}, P_{h, \varepsilon}\right) \geq a\left(P_{h}, P_{h}\right) \geq 0,
$$

which implies that

$$
a\left(P_{h}, W_{h}-P_{h}\right) \geq\left(f_{1}, W_{h}-P_{h}\right), \quad W_{h} \in B \subset H_{h}^{2}\left(\Omega_{h}\right) .
$$

Now we shall show that (13) holds for all $\omega_{h} \in H_{h}^{2}\left(\Omega_{h}\right)$. Indeed,

$$
\omega_{h}=\sum_{i} \alpha_{i} \omega_{i}, \quad \alpha_{i} \geq 0, \quad \omega_{i} \in B,
$$

so that

$$
\begin{gathered}
a\left(P_{h}, \omega_{h}-P_{h}\right)=a\left(P_{h},\left(\sum_{i} \alpha_{i} \omega_{i}\right)-P_{h}\right)=\sum_{i} \alpha_{i} a\left(P_{h}, \omega_{i}-P_{h}\right) \\
\geq \sum_{i} \alpha_{i}\left(f_{1}, \omega_{i}-P_{h}\right),
\end{gathered}
$$

since the $\alpha_{i}$ 's are positive, and (13) is valid for all $\omega_{i}$ 's. Thus

$$
a\left(P_{h}, \omega_{h}-P_{h}\right) \geq\left(f_{1}, \omega_{h}-P_{h}\right) ; \quad \omega_{h} \in H_{h}^{2}\left(\Omega_{h}\right),
$$

which shows that $P_{h}$ is a solution of the inequality (4).

Since $P_{h} \geq 0$, that is $P_{h} \in H_{h}^{2}\left(\Omega_{h}\right),(14)$ is a variational inequality; the unique solution (in $K_{1}$ ) of (14) minimises

$$
I\left(P_{h}\right)=1 / 2 \int_{\Omega_{h}}\left|\nabla P_{h}\right|^{2} d x-\int_{D_{h}}\left(f_{1}, P_{h}\right) d x,
$$

on the convex set $K_{1}$.

Thus, we can estimate $P_{h}$ by actually performing the minimisation (15). In the case of Theorem 2, we can see that the functional I is the limit as $\varepsilon$ tends to zero of

$$
I_{\varepsilon}\left(P_{h, \varepsilon}\right)=1 / 2 \int_{\Omega_{h}}\left(\left|\nabla P_{h, \varepsilon}\right|^{2}+F^{\prime}\left(\varphi_{h, \varepsilon}\right) P_{h, \varepsilon}^{2}\right) d x-\int_{D_{h}} f_{1} P_{h, \varepsilon} d x
$$


(for more detail see Butt [2]); this fact in effect can be proved by Theorem 2.

\section{References}

[1] F. Angrand, "Numerical method for optimal shape design in aerodynamics", 3 Cycle Thesis, University of Paris 6, 1980.

[2] R. Butt, "Optimal shape design for differential inequalities", Ph. D. Thesis, Leeds University, U.K., 1988.

[3] P. Ciarlet, The finite element method (North Holland, Amsterdam, 1979).

[4] R. Glowinski, J. L. Lions and R. Tremolieres, Theory of variational inequalities (North Holland, Amsterdam, 1981).

[5] J. L. Lions, "Some topics on variational inequalities and applications", in New developments in differential equations (ed. W. Eckaus), (North-Holland Publishing Company, 1976) 1-38.

[6] O. Pironneau, Optimal shape design for elliptic systems (Springer-Verlag, New York, 1984). 